\title{
Interactions Between Anti-Epileptic Drugs and Contraceptives: A Review
}

\author{
BHARAT SHARMA ${ }^{1}$ PARUL SAKSHI*2
}

Around half of the pregnancies in women with epilepsy are unplanned and the choice of contraceptive method in epileptic women is important, since it requires considering their possible pharmacological interactions with certain types of anti-epileptic drugs. Drugs from this class which induce hepatic enzyme activity may alter the metabolism of most hormonal methods of contraception, and this may affect their contraceptive efficacy. Hormonal contraception is regarded as highly effective, but its interaction with anti-epileptics may accelerate the metabolism of the latter with the consequent risk of failure, reduction of plasma concentration predisposing to seizures, risk of unwanted pregnancies, abortions, teratogenicity, maternal or fetal complications and difficulty in the management of epilepsy during pregnancy. In case of prescribing both medications, the combined use with a barrier method should be considered. Family planning counselling at the first visit has been shown to influence the choice of the contraceptive method. In conclusion, the different therapeutic options should be analyzed together with the epileptic patients in order to achieve and optimize the best goal for each one This article reviews these issues and offers practical recommendations for the management of contraception in epileptic patients.

KEYWORDS: Epilepsy, Contraception, Anti-Seizure Drugs, Pregnancy

\section{INTRODUCTION}

Epilepsy is a chronic neurological disease, characterized by recurrent seizures resulting from abnormal neuronal discharge in the central nervous system. Around 65 million subjects are affected by it globally. ${ }^{1}$ Changes in seizure patterns have been reported in women during puberty, the menstrual cycle, pregnancy and menopause, related to hormone steroid levels. ${ }^{2}$ Estrogens are generally considered proconvulsants via the glutamate receptor excitatory pathway, while progesterone and its metabolites are anticonvulsants via the inhibitory effect via the neuronal membrane receptor, enhancing GABA-A mediated chloride conductance. ${ }^{2-4}$ The seizure fluctuations that occur during the menstrual cycle are responsible for one third of cases and are defined as catamenial epilepsy. ${ }^{2-4}$ The drug-drug interaction between anticonvulsant drugs and contraceptives was first suggested in the early $197 \mathrm{O}_{\mathrm{s} .} .5$ Therefore, contraception management in these women is critical because of the potential maternal fetal risk due to failure in the choice of method. ${ }^{1,6,7}$ There may also be difficulty managing seizure activity, caused by poor adherence to the drug treatment or physiological changes in the pharmacokinetics of the anticonvulsant medication during pregnancy. ${ }^{8}$ There are currently different methods of birth control available: non-hormonal contraception such as: copper intrauterine devices, barrier methods, surgical and hormonal, transdermal patches subdermal implants, vaginal rings, and finally levonorgestrelreleasing intrauterine devices. Another form of contraceptive use is emergency hormonal contraception. The failure rate is approximately $1 \%$ in healthy women, but $3-6 \%$ in women with epilepsy. This occurs despite the fact that fertility in this group of patients is reduced by one third, either due to social reasons or organic reasons. ${ }^{9}$ The estrogen component found in most formulations of oral combined contraceptives is ethinylestradiol.

More than $30 \%$ of the ethinylestradiol undergoes metabolization in the intestinal wall, mainly by sulfotransferase - dependent conjugation. Subsequently its biotransformation continues in the liver catalyzed by cytochrome $\mathrm{P} 450$ enzyme complex enzymes, uridine diphosphate glucuronyltransferase ${ }_{1} \mathrm{~A} 1$ and sulfotransferase-dependent conjugation, and finally, it is hydroxylated to inactive metabolites predominantly by cytochrome $\mathrm{P}_{450} 3 \mathrm{~A}_{4}$. These hydroxylated and methylated metabolites are present free and conjugated to glucuronides and sulfate. 9 Glucuronidation is inhibited by valproate and induced by phenobarbital, phenytoin, carbamazepine, oxcarbazepine and high dose topiramate, accelerating the metabolism of ethinylestradiol. ${ }^{10}$ Ethinylestradiol can also induce the enzymes uridine diphosphate 
glucuronyl transferase, affecting drugs that are primarily metabolized by this pathway, such as lamotrigine. ${ }^{9}$ Similar to ethinylestradiol, the metabolism of the progestogen component also involves cytochrome $\mathrm{P} 450$ by hydroxylation, reduction, deacetylation and finally sulfation and glucuronidation. ${ }^{9}$ Its metabolism may be accelerated if combined with carbamazepine, oxcarbazepine, lamotrigine, phenytoin and phenobarbital. ${ }^{10}$ The firstgeneration of anticonvulsant drugs includes phenobarbital, phenytoin, valproate, diazepam, clonazepam, carbamazepine, ethosuximide and primidone." The second-generation drugs include felbamate, gabapentin, clobazam, lamotrigine, levetiracetam, pregabalin, tiagabine, topiramate, vigabatrin and zonisamide. ${ }^{12}$

Finally, with the aim of obtaining minimal or no adverse effects with anticonvulsant properties, the door was opened to the third generation: brivaracetam, eslicarbazepine acetate, lacosamide, rufinamide, stiripentol, perampanel. ${ }^{13}$ Based on their mechanism of hepatic metabolism, anticonvulsant drugs may be classified as $\mathrm{P} 450$ enzyme-inducing and non-enzyme-inducing antiepileptic drugs. $\mathrm{P}_{450}$ system enzyme inducers include phenobarbital, phenytoin, carbamazepine, oxcarbazepine, eslicarbazepine, felbamate, primidone, rufinamide, lacosamide, and topiramate (at dose greater than 200 $\mathrm{mg} /$ day). These drugs induce the cytochrome $\mathrm{P}_{450}$ enzymes from the liver, being able to accelerate the metabolism of combined or progestogen-only oral contraceptives and progestogen implants (subdermal) reducing their effectiveness. ${ }^{2,9}$

Oxcarbazepine has a low enzyme induction potential, but unfortunately exerts a similar effect to carbamazepine on hormonal contraceptives.9,14 Therefore, if oral contraception is indicated, use in combination with a barrier method such as condom or vaginal diaphragm is suggested. ${ }^{15,16}$ These anticonvulsant drugs do not alter the efficacy of depot medroxyprogesterone acetate injection, copper intrauterine device or levonorgestrel release, therefore these may be another option. ${ }^{16}$ In turn, these drugs used as monotherapy increase sex hormone-binding globulin levels, both in males and females, resulting in decreased levels of testosterone and bioavailable estradiol, increasing its metabolism with subsequent reduction in its plasma concentration. ${ }^{15}$ With some drugs, the extent of enzyme induction may be dosedependent, difficult to quantify and subject to large variation between individuals, as occurs with topiramate. No enzyme inducers of the $\mathrm{P} 450$ system are namely levetiracetam, gabapentin, topiramate (at doses below $200 \mathrm{mg}$ per day), zonisamide, clobazam, tiagabine, brivaracetam and vigabatrin. This group does not affect contraceptive efficacy, so epileptic women can choose any method of contraception or emergency contraception. ${ }^{16}$ The drug lamotrigine has minimal effects on the efficacy of combined contraceptives, however, the use of this type of contraception increases clearance, reducing the plasma concentration of lamotrigine by 45 to $60 \%$ and predisposing to epilepsy seizures. If this is the option chosen by the epileptic woman, the dose of lamotrigine should be increased and to reduce its toxicity. Along with levetiracetam, it is recommended during pregnancy because of its low risk of teratogenicity. Enzyme inhibitor anticonvulsant drugs valproate is used as a first option in many women with generalized epilepsy and in unclassified epilepsy, achieving control with low doses. It is highly teratogenic in a dose-dependent manner, and genetic factors and individual susceptibility may be involved. It has been described to act as an antagonist of folic acid. ${ }^{17}$ A significantly lower intelligence quotient was detected in children of women treated with valproate, as well as a lower verbal, nonverbal and spatial response and the need for an 8-fold higher education intervention. Intrauterine exposure to this drug is associated with an increased risk of autism spectrum disorders, hyperactivity, dyspraxia and attention deficit disorder. ${ }^{8}$ Stiripentol inhibits cytochrome $\mathrm{P}_{450}$ $3 \mathrm{~A}_{4}$. There are no publications on the interaction of this drug with contraceptives, so its action on this pathway is assumed to be mild. ${ }^{14}$

Literature suggests increase in risk of seizures during contraception. A birth control registry was conducted in 1144 epileptic women, aged 18 to 47 years to find out the impact of contraceptive methods on seizures in different antiepileptic drug category users. For each category of anticonvulsant drugs, they reported an approximately 4.5 -fold increased risk of seizures while taking hormonal contraception versus nonhormonal. ${ }^{3}$ Within specific types of hormonal contraception, a $68 \%$ higher incidence of seizures was reported with hormonal patches when compared to users of combined oral contraceptives, probably due to higher ethinylestradiol levels, because gastrointestinal absorption and the first step of hepatic metabolism are avoided. ${ }^{3}$ And those who used the progestogen-only contraceptive pill had a $62 \%$ increase in seizures. This could be due to the low progestin content; whose contraceptive action is more 
on cervical mucus than on hormone suppression. Within monotherapy, lamotrigine and valproate presented increased attacks when combined with hormonal contraception. ${ }^{1,3}$ The precise mechanism by which hormonal based contraceptives may increase the risk of epileptic seizures remains unknown, as there is little evidence regarding the neuroactive properties of the synthetic steroids of contraceptives.

Three potential pathways have been proposed: by classic genomic action estrogens in the central nervous system binds to specific receptors, dimerize and modify neuronal gene expression. Due to nongenomic effects through membrane receptor activation, regardless of the pathway, they can generate changes in neuronal structure, plasticity, direct excitatory effects on the neuronal membrane and postsynaptic regulation of different neurotransmitters. Finally, indirect effects due to alteration of the metabolism of anticonvulsant drugs via induction of the hepatic cytochrome P450 enzyme system, leading to drug-drug interactions and thus reducing therapeutic efficacy when administered together. ${ }^{1,4}$ A retrospective, observational cohort study with case-control analysis was described, conducted from the United Kingdom Health Improvement Network database with 2,201 epileptic women aged 20 to 44 years. This study showed no association between combined oral contraceptives or the progestogen-only hormonal pill and the risk. of epilepsy. However, the limitation of this study was low utilization of contraceptives. ${ }^{18}$ Another study on epileptic women reported that $63 \%$ had received family planning information, however, $54 \%$ reported a previous pregnancy. About half of these women were not using contraception and among those who were users, $11 \%$ were at risk of potential interaction between oral contraceptives and enzyme-inducing anti-epileptic drugs. $80 \%$ received folic acid during pregnancy, although most started supplementation in the second trimester of pregnancy. ${ }^{19}$

Another study reports that $65 \%$ of the women had had at least one unwanted pregnancy, 30.3\% of them did not use a contraceptive method considered highly effective, correlated to lower income, younger age and less access to health insurance. ${ }^{20} 14.5 \%$ used hormonal contraception in combination with enzyme-inducing anti-epileptic drugs that could compromise contraceptive efficacy and 50\% did not take folic acid before or during pregnancy. Among users with reversible contraceptive methods, $40.6 \%$ discontinued it. When compared with the various anti-epileptic drugs, combination with enzyme inducers most commonly caused the discontinuation of contraceptive methods. Only $25.4 \%$ of epileptic women had consulted with their neurologist regarding the choice of contraceptive method. The most common reasons for discontinuation were concern about reliability (13.9\%), menstrual problems (13.5\%) and increased seizures (8.6\%). ${ }^{21}$ A study by Espinera et al. showed how counselling with the epileptologist may influence the choice of contraceptive method. ${ }^{22}$ Patients who did not desire to get pregnant, when advised about the intrauterine device as a first choice of birth control, were more willing to change to this contraceptive method and were more successful if this topic was addressed at the first neurological consultation. Another survey of neurologists from Brazil reported that $76 \%$ conducted family planning counseling, $81 \%$ recommended the use of copper intrauterine device, $60 \%$ simultaneously prescribed anticonvulsant drugs and hormonal contraception. Although $93 \%$ were aware that it could contraindicate the use of some contraceptives, they were unaware of the specific mechanism of interaction between both drugs. ${ }^{23}$

During pregnancy, most epileptic women experience improvement of epilepsy, especially those treated with monotherapy and even remain seizure-free, as demonstrated by the European and International Registry of Antiepileptic Drugs and Pregnancy. ${ }^{24}$ Only $2.6 \%$ of pregnant women needed to increase the dose or add a second anticonvulsant drug. In addition, there is a $4.9 \%$ risk of malformations in children of women receiving anticonvulsants during pregnancy versus $2.2 \%$ in the general healthy population. The use of an anticonvulsant drug in monotherapy doubles the risk, while combination therapy triples it. Prior folic acid supplementation is associated with neurodevelopmental benefits, where children born to pharmacologically treated epileptic mothers, without prior exposure to folic acid, are at increased risk of autistic traits, and this is inversely associated with maternal plasma folate concentration and folic acid dose. Therefore, when planning to get pregnant, it is recommended to start taking folic acid, at least until the first trimester of pregnancy.

\section{CONCLUSION}

Epilepsy in women is influenced by physiological variations in the secretion of sex hormones during the menstrual cycle in reproductive life. Therefore, it is important to know aspects of treatment that are 
specific at this stage, such as the combination of anticonvulsant drugs and contraceptives, menstrual cycle-related seizures and their adverse effects. It is important to discuss with epileptic patients regarding the adverse effects, preference, efficacy, risks and benefits of the different methods of contraception, as well as the possible interactions with some anticonvulsant drugs in order to optimize the best method. With advancement in the pharmaceutical industry, we have a greater selection of anticonvulsant drugs, with better efficacy and tolerance and fewer drug interactions. The concept of monotherapy or rational combination therapy is important in women who are sexually active and who do not want to conceive, so they should be oriented on the most effective form of contraception.

\section{REFERENCES}

1.Reddy DS. Do oral contraceptives increase epileptic seizures? Expert Rev Neurother. 2017; 17: 129-34.

2. Bangar S, Shastri A, El-Sayeh H, Cavanna AE. Women with epilepsy: clinically relevant issues. Funct Neurol 2016; 31: 127-34.

3. Herzog AG, Mandle HB, Cahill KE, Fowler KM, Hauser WA. Differential impact of contraceptive methods on seizures varies by antiepileptic drug category: findings of Epilepsy Birth. Epilepsy Behav 2016; 60: 112-7.

4. Tauboll E, Sveberg L, Svalheim S. Interactions between hormones and epilepsy. Seizure 2015; 28: 3-11. 5. Sabers A. Pharmacokinetic interactions between contraceptives and antiepileptic drugs. Seizure 2008; 17: 141-4.

6. Herzog AG, Mandle HB, Cahill KE, Fouler KM, Hauser WA. Predictors of unintended pregnancy in women with epilepsy. Neurology 2017; 88: 728-33.

7. Thomas SV. Controversies in contraception for women with epilepsy. Ann Ind Acad Neurol 2015; 18: 278-83.

8. Stephen LJ, Harden C, Tomson T, Brodie MJ. Management of epilepsy in women. Lancet Neurol 2019; 18: 481-91.

9. Reimers A, Brodtkord E, Sabers A. Interactions between hormonal contraception and antiepileptic drugs clinical and mechanistic considerations. Seizure 2015; 28: 66-70.

10. Bosak M, Cyranka k, Slowik A. Hormonal contraception in patients with epilepsy. Ginekol Pol 2019; 90: 61-5.
11. Brodie MJ. Antiepileptic drug therapy the story so far. Seizure 2010; 19: 650-5.

12. Bialer M. New antiepileptic drugs that are second generation to existing antiepileptic drugs. Expert Opin Investig Drugs 2006; 15: 637-47.

13. Vidaurre J, Herbst J. New antiepileptic drugs. Medicina (B Aires) 2019; 79: 48-53.

14. Stefanović S, Janković SM, Novaković M, Milosavljević. Pharmacodynamics and common drugdrug interactions of the third-generation antiepileptic drugs. Expert Opin Drug Metab Toxicol 2018; 14: 1539.

15. Burgos NS, Parra MA. Epilepsia y anticonceptivos. Rev Chil Obstet Ginecol 2004; 69: 470-5.

16. Gooneratne IK, Wimalaratna R, Ranaweera AKP, Wimalaratna S. Contraception advice for women with epilepsy. BMJ 2017; 357-60.

17. Herzog AG, Mac Eacherm DB, Mandle Hb, Cahill KE, Fowler KM, Davis AR. Folic acid use by women with epilepsy: Findings of the Epilepsy Birth. Epilepsy Behav 2017; 72: 156-6o.

18. Beier CP, García Rodríguez LA, Sáez ME, Gaist D, González- Pérez A. Hormonal contraception is not associated with increased risk for seizures in the general population: results from a cohort study using The Health Improvement Network. Eur J Clin Pharmacol 2018; 74: 1175-80.

19. Halani S, Tshering L, Bui E, et al. For the Brutan Epilepsy Project. Contraception, pregnancy and peripartum experiences among women with epilepsy in Bhutan. Epilepsy Res 2017; 138: 116-23.

20. Herzog AG, Mandle HB, Cahill KE, Fowler KM, Hauser WA. Contraceptive practices of women with epilepsy: Findings of the epilepsy birth control registry. Epilepsia 2016; 57:630-7.

21. Mandle HB, Cahill KE, Fowller KM, Hauser WA, Davis AR, Herzog AG. Reason for discontinuation of reversible contraceptive methods by women with epilepsy. Epilepsia 2017; 58: 907-11.

22. Espinera AR, Gavvala J, Bellinski I, et al. Counseling by epileptologists affects contraceptive choices of women with epilepsy. Epilepsy Behav 2016; 65: 1-6.

23. Suto HS, Braga GC, Scarpellini GR, et al. Neurologist knowledge about interactions between antiepileptic drugs and contraceptive methods. Int J Gynaecol Obstet 2016; 134: 264-7.

24. Battino D, Tomson T, Bonizzoni E, et al. European Study group. Epilepsy 2013; 54: 1621-7. 


\section{Cite this article as:}

Sharma B, Sakshi P. Interactions Between Anti-Epileptic Drugs and Contraceptives: A

Source of support: Nil, Conflict of interest: None declared

Review. Int Healthc Res J. 2021;4(11):RV14-RV18. https://doi.org/10.26440/IHRJ/0501.04409

AUTHOR AFFILIATIONS: (*Corresponding Author)

1. MBBS, Senior Lecturer, General Medicine, Shri Sukhmani Dental College and Hospital, Dera Bassi, Mohali, Punjab, India

2. Associate Physician, Adult Medicine, Kaiser Permanente San Jose Medical Center, 250 Hospital Pkwy, San Jose, CA 95119

Contact corresponding author at: parulnijhawan2[at]yahoo[dot]com 